\title{
SZEGÖ COORDINATES, QUADRATURE DOMAINS, AND DOUBLE QUADRATURE DOMAINS
}

\author{
STEVEN R. BELL, BJÖRN GUSTAFSSON, AND ZACHARY A. SYLVAN
}

\begin{abstract}
We define Szegö coordinates on a finitely connected smoothly bounded planar domain which effect a holomorphic change of coordinates on the domain that can be as close to the identity as desired and which convert the domain to a quadrature domain with respect to boundary arc length. When these Szegö coordinates coincide with Bergman coordinates, the result is a double quadrature domain with respect to both area and arc length. We enumerate a host of interesting and useful properties that such double quadrature domains possess, and we show that such domains are in fact dense in the realm of bounded $C^{\infty}$-smooth finitely connected domains.
\end{abstract}

\section{INTRODUCTION}

The unit disc $D_{1}(0)$ is the quintessential example of a double quadrature domain. Holomorphic functions $h$ on the disc satisfy two quadrature identities:

$$
\pi h(0)=\iint_{D_{1}(0)} h d A \quad \text { and } \quad 2 \pi h(0)=\int_{b D_{1}(0)} h(z) d s
$$

whenever these integrals make sense. We will show in this paper that double quadrature domains are rather commonplace among smoothly bounded domains in the plane.

A bounded domain $\Omega$ in the complex plane is a quadrature domain with respect to area measure if there exist finitely many points $\left\{w_{j}\right\}_{j=1}^{N}$ in the domain and non-negative integers $n_{j}$ such that complex numbers $c_{j k}$ exist satisfying

$$
\iint_{\Omega} h d A=\sum_{j=1}^{N} \sum_{k=0}^{n_{j}} c_{j k} h^{(k)}\left(w_{j}\right)
$$

for every function $h$ in the Bergman space $H^{2}(\Omega)$ of square integrable holomorphic functions on $\Omega$. Here, $d A$ denotes Lebesgue area measure. We shall say that such a domain is an area quadrature domain. Aharonov and Shapiro [1] proved that such domains have piecewise real-analytic boundaries (see also [14]).

A bounded domain $\Omega$ bounded by finitely many non-intersecting piecewise $C^{1}$-smooth Jordan curves is a quadrature domain with respect to boundary arc

1991 Mathematics Subject Classification. 30C35.

Key words and phrases. Bergman kernel, Poisson kernel, conformal mapping. 
length measure if there exist finitely many points $\left\{w_{j}\right\}_{j=1}^{N}$ in the domain and non-negative integers $n_{j}$ such that complex numbers $c_{j k}$ exist satisfying

$$
\int_{b \Omega} h d s=\sum_{j=1}^{N} \sum_{k=0}^{n_{j}} c_{j k} h^{(k)}\left(w_{j}\right)
$$

for every function $h$ in the Hardy space $H^{2}(b \Omega)$ of holomorphic functions with $L^{2}$ boundary values. Here, $d s$ denotes the boundary arc length measure. We shall say that such a domain is a boundary arc length quadrature domain. Boundary arc length quadrature domains have $C^{\infty}$-smooth real-analytic boundaries (see [15], where it is also shown that the boundary regularity assumptions can be greatly relaxed).

After some pioneering work of Avci [2] and Shapiro and Ullemar [21], the second author [15] studied planar quadrature domains with respect to boundary arc length from the point of view of half-order differentials and Riemann surface theory; he characterized such domains and showed that they are dense in the category of bounded domains in the plane bounded by finitely many nonintersecting Jordan curves. Here, we shall reframe these results in more elementary terms and explain how to view boundary arc length quadrature domains in terms of Szegö coordinates, which are the analogue of Bergman coordinates as developed in [9, 10, 11]. Because our results are most interesting in the category of $C^{\infty}$-smoothly bounded domains, we shall take the royal road and restrict our attention to domains of this form.

For more information about the state of research on quadrature domains, see [17] and the volume [13] that holds it, and [20]. We also remark that it is possible to study quadrature domains with respect to harmonic functions in the plane, and to prove density results in this context (see [16] and Sakai [19]).

Suppose that $\Omega$ is a bounded domain bounded by $n$ non-intersecting $C^{\infty}$ smooth Jordan curves. Let $A^{\infty}(\Omega)$ denote the subspace of $C^{\infty}(\bar{\Omega})$ consisting of holomorphic functions. Let $K(z, w)$ denote the Bergman kernel associated to $\Omega$ and let $S(z, w)$ denote the Szegö kernel. Let $K^{0}(z, w)$ also denote $K(z, w)$ and let $K^{m}(z, w)=\frac{\partial^{m}}{\partial \bar{w}^{m}} K(z, w)$. As in [11], we define the Bergman span associated to $\Omega$ to be the complex linear span $\mathcal{K}$ of all functions $h(z)$ of $z$ of the form $K^{m}(z, a)$ as a ranges over $\Omega$ and $m$ ranges over all non-negative integers. If $U$ is an open subset of $\Omega$, let $\mathcal{K}_{U}$ denote the complex linear span of functions of $z$ from $\{K(z, a): a \in U\}$, and given a point $a$ in $\Omega$, let $\mathcal{K}_{a}$ denote the complex linear span of functions of $z$ from $\left\{K^{m}(z, a): m=0,1,2, \ldots\right\}$. Similarly, define the $S z$ zego span to be the complex linear span $\mathcal{S}$ of all functions $h(z)$ of $z$ of the form $S^{m}(z, a)$ as $a$ ranges over $\Omega$ and $m$ ranges over all non-negative integers, and define $\mathcal{S}_{U}$ and $\mathcal{S}_{a}$ in the analogous way.

We now list two theorems that will serve as the heart of the paper. Note that if $f: \Omega_{1} \rightarrow \Omega_{2}$ is a biholomorphic mapping between finitely connected domains in the plane, then it is well known that there is a holomorphic branch of $\sqrt{f^{\prime}}$ on $\Omega_{1}$. 
Theorem 1.1. Suppose $\Omega_{1}$ and $\Omega_{2}$ are bounded domains in the plane bounded by finitely many non-intersecting $C^{\infty}$-smooth Jordan curves. Suppose further that $f: \Omega_{1} \rightarrow \Omega_{2}$ is a biholomorphic mapping. Then $\Omega_{2}$ is a boundary arc length quadrature domain if and only if $f^{\prime}$ is equal to the square of an element of the Szegö span associated to $\Omega_{1}$, or equivalently, if and only if $\sqrt{f^{\prime}}$ is in the Szegö span.

We shall call a biholomorphic mapping $f$ as given in Theorem 1.1 such that $\sqrt{f^{\prime}}$ is in the Szegö span a Szegö coordinate. This is a direct analogue of the definition of Bergman coordinate in [11] where a biholomorphic map $f$ is called a Bergman coordinate if and only if $f^{\prime}$ is in the Bergman span.

The next theorem allows us to use Theorem 1.1 to approximate domains by boundary arc length quadrature domains.

Theorem 1.2. Suppose that $\Omega$ is a bounded domain bounded by finitely many non-intersecting $C^{\infty}$-smooth Jordan curves. Then the Szego" span $\mathcal{S}$ associated to $\Omega$ is dense in $A^{\infty}(\Omega)$. Furthermore, $\mathcal{S}_{U}$ is dense in $A^{\infty}(\Omega)$ for any open subset $U$ of $\Omega$, and $\mathcal{S}_{a}$ is dense in $A^{\infty}(\Omega)$ for any point $a \in \Omega$.

Theorem 1.2 was proved in [4].

Theorems 1.1 and 1.2 and results of [11] and [15] can be combined to obtain the following results. The next theorem shows that boundary arc length quadrature domains are dense in the realm of bounded finitely connected smoothly bounded planar domains.

Theorem 1.3. Suppose $\Omega$ is a bounded domain bounded by finitely many nonintersecting $C^{\infty}$-smooth Jordan curves. There is a biholomorphic mapping $f$ which is a Szego" coordinate as close to the identity in $C^{\infty}(\bar{\Omega})$ as we please. Hence, $f(\Omega)$ is a boundary arc length quadrature domain which is $C^{\infty}$ close to $\Omega$.

The proof of Theorem 1.3 was sketched in [8, p. 285-6]. We shall flesh out the argument here in $\$ 4$ to make this paper more comprehensible and because we will need the fine details in order to generalize the result to double quadrature domains.

Theorem 1.4. Suppose $\Omega$ is a bounded n-connected domain bounded by $n$ nonintersecting $C^{\infty}$-smooth Jordan curves. There is a biholomorphic mapping $f$ which is both a Szego" coordinate and a Bergman coordinate as close to the identity in $C^{\infty}(\bar{\Omega})$ as we please. Hence, $f(\Omega)$ is both a boundary arc length quadrature domain and an area quadrature domain, i.e., a double quadrature domain, which is $C^{\infty}$ close to $\Omega$.

We will prove Theorem 1.4 in $\$ 3$ in case the domain is simply connected and prove the general case in $\$ 5$.

The Riemann mapping theorem says that every simply connected domain can be mapped to a special double quadrature domain, the unit disc. Our results can 
be thought of as a generalization, or maybe even an improvement. Every finitely connected domain in the plane can be mapped to a nearby double quadrature domain.

Double quadrature domains have many remarkable properties in common with the unit disc, some of which we enumerate here.

- The boundary is given by finitely many non-intersecting real algebraic curves which are $C^{\infty}$-smooth and real analytic. (It is shown in [14] that the boundary is essentially given by the zero set of an irreducible polynomial $P(z, w)$ on $\mathbb{C}^{2}$. In fact, the boundary is equal to $\{z: P(z, \bar{z})=0\}$ minus perhaps finitely many points, and $P(z, w)$ must have a a certain special form.)

- The complex polynomials belong to the Bergman span.

- The complex polynomials belong to the Szegő span. (In fact, this property characterizes double quadrature domains. See Theorem 6.2.)

- The complex unit tangent vector function $T(z)$ is a rational function of $z$ and $\bar{z}$ for $z$ in the boundary (which of course is equivalent to being a rational function of $\operatorname{Re} z$ and $\operatorname{Im} z)$. The function $T(z)$ is also the restriction to the boundary of a meromorphic function on the double without poles on the boundary. This means that $T(z)$ can be extended to the domain as either a meromorphic function with no poles on the boundary, or an anti-meromorphic function with no poles on the boundary. (A more precise statement of this known fact can be found in Theorem 6.1.)

- The Schwarz function $S(z)$, which exists and extends to be analytic on a neighborhood of the boundary by virtue of the real analyticity of the boundary, also extends to be meromorphic on the domain. It has no poles on the boundary, and it is an algebraic function. Because $S(z)=\bar{z}$ on the boundary, both $z$ and $S(z)$ extend meromorphically to the double of the domain. It was noted in [14] that all the meromorphic functions on the double are generated by the extensions of $z$ and $S(z)$ to the double.

- Both the Bergman kernel $K(z, w)$ and the Szegö kernel $S(z, w)$ are rational functions of $z, S(z), \bar{w}$, and $\overline{S(w)}$. Hence, they both extend to the double cross the double as functions which are meromorphic in $z$ and anti-meromorphic in $w$. If the domain is multiply connected, the two kernels are rational functions of $f_{1}(z), f_{2}(z), \overline{f_{1}(w)}$, and $\overline{f_{2}(w)}$, where $f_{1}$ and $f_{2}$ are two Ahlfors maps associated to two generic points in the domain. In the simply connected case, the kernels are even more elementary (see below).

- Both the Bergman and Szegö kernels are rational functions of $z, \bar{z}, w$, and $\bar{w}$ when $z$ and $w$ are restricted to the boundary.

- The Kerzman-Stein kernel $A(z, w)$ is a rational function of $z, \bar{z}, w$, and $\bar{w}$ for $z$ and $w$ on the boundary.

If the domain is a simply connected double quadrature domain, then even more can be said. Let $f$ denote a Riemann map associated to a point in the domain (which maps the domain one-to-one onto the unit disc). 
- $f$ extends to the double because $f(z)=1 / \overline{f(z)}$ on the boundary. Hence, it follows that $f(z)$ is a rational function of $z$ and $S(z)$, and so it is a rational function of $z$ and $\bar{z}$ on the boundary. It is also algebraic. Since the extension of $f$ to the double generates the meromorphic functions on the double, it also

follows that $z$ and $S(z)$ are rational functions of $f(z)$. (This is another way to deduce that $f$ has a rational inverse, a well know fact about simply connected quadrature domains with respect to area.)

- The Bergman kernel $K(z, w)$ and the Szegő kernel $S(z, w)$ are rational functions of $f(z)$ and $\overline{f(w)}$.

- The Poisson kernel $p(z, w)$ is the real part of a function that is rational in $z$ and $S(z)$, and $w$ and $\bar{w}$ (for $z$ in the domain and $w$ on the boundary). It is also the real part of a rational function of $f(z)$ and $w$ and $\bar{w}$. It is even the real part of a rational function of $f(z)$ and $f(w)$.

We shall explain these results in $₫ 6$. In $\$ 7$, we reveal a connection between the Schwarz function, quadrature domains, and proper holomorphic mappings onto the unit disc. We prove, for example, that a domain is an area quadrature domain if and only if, on the boundary, $z / \bar{z}$ is equal to the boundary values of a quotient of proper holomorphic mappings to the unit disc (Theorem 7.1). A smooth domain is an arc length quadrature domain if and only if the complex unit tangent vector function on the boundary is equal to the boundary values of a quotient of proper holomorphic mappings to the unit disc (Theorem [7.2).

\section{Proof of TheOrem 1.1}

Suppose that $f: \Omega_{1} \rightarrow \Omega_{2}$ is a biholomorphic mapping between bounded domains in the plane bounded by finitely many non-intersecting $C^{\infty}$-smooth Jordan curves. Let $F=f^{-1}$. It is well known that a holomorphic square root of $f^{\prime}$ exists on $\Omega_{1}$ and a square root of $F^{\prime}$ exists on $\Omega_{2}$. Since $f^{\prime}$ and $F^{\prime}$ extend $C^{\infty}$-smoothly to the respective boundaries and are non-vanishing there, the same is true of their square roots. Notice that the change of variables formula yields that

$$
\int_{b \Omega_{1}}\left|f^{\prime}\right|(u \circ f) d s=\int_{b \Omega_{2}} u d s
$$

when $u$ is in the Hardy space $H^{2}\left(b \Omega_{2}\right)$. Let

$$
\langle u, v\rangle_{j}=\int_{b \Omega_{j}} u \bar{v} d s
$$

denote the Hardy space inner product on $\Omega_{j}, j=1,2$. The last identity can be written

$$
\left\langle\sqrt{f^{\prime}}(u \circ f), \sqrt{f^{\prime}}\right\rangle_{1}=\langle u, 1\rangle_{2} .
$$

It is easy to verify that if $u \in L^{2}\left(b \Omega_{2}\right)$ and $v \in L^{2}\left(b \Omega_{1}\right)$, then

$$
\left\langle\sqrt{f^{\prime}}(u \circ f), v\right\rangle_{1}=\left\langle u, \sqrt{F^{\prime}}(v \circ F)\right\rangle_{2} .
$$


To prove the first half of Theorem 1.1, assume that $\sqrt{f^{\prime}}$ is in the Szegö span associated to $\Omega_{1}$. If $h$ is an element of the Hardy space $H^{2}\left(b \Omega_{2}\right)$, then

$$
\int_{b \Omega_{2}} h d s=\int_{b \Omega_{1}}\left|f^{\prime}\right|(h \circ f) d s=\left\langle\sqrt{f^{\prime}}(h \circ f), \sqrt{f^{\prime}}\right\rangle_{1},
$$

and if $\sqrt{f^{\prime}}$ is in the Szegö span, then this last inner product yields a finite linear combination of values of $\sqrt{f^{\prime}}(h \circ f)$ and its derivatives at finitely many points in $\Omega_{1}$, which reduces to a finite linear combination of values of $h$ and its derivatives at finitely many points in $\Omega_{2}$. This shows that $\Omega_{2}$ is a boundary arc length quadrature domain.

To prove the converse, suppose that $\Omega_{2}$ is a boundary arc length quadrature domain. Then, given any $g \in H^{2}\left(b \Omega_{1}\right)$,

$$
\left\langle g, \sqrt{f^{\prime}}\right\rangle_{1}=\left\langle\sqrt{F^{\prime}}(g \circ F), 1\right\rangle_{2},
$$

and since $\Omega_{2}$ is a boundary arc length quadrature domain, this last inner product yields a finite linear combination of values of $\sqrt{F^{\prime}}(g \circ F)$ and its derivatives at finitely many points in $\Omega_{2}$, which reduces to a finite linear combination of values of $g$ and its derivatives at finitely many points in $\Omega_{1}$. Thus, $\sqrt{f^{\prime}}$ has the same effect as an element of the Szegö span when paired against a function $g$ in the Hardy space. It follows that $\sqrt{f^{\prime}}$ must in fact be equal to that element in the Szegő span.

\section{Density of quadrature Domains in the Simply CONneCted CASE}

In this section, we give two proofs that boundary arc length quadrature domains are dense in the realm of bounded $C^{\infty}$-smoothly bounded simply connected domains, and that double quadrature domains are dense as well. The first proof introduces techniques that we will generalize to the multiply connected setting. The second proof is so easy, natural, and short that one could get the wrong impression that the multiply connected generalization should be easy and straightforward.

Suppose that $\Omega$ is a bounded simply connected domain in the plane bounded by a $C^{\infty}$-smooth Jordan curve. We may now construct a boundary arc length quadrature domain close to $\Omega$ as follows. By Theorem 1.2, there exists a function $h$ in the Szegö span that is close to the constant function 1 in $A^{\infty}(\Omega)$. (Of course, we take $h$ close enough to 1 so as to be non-vanishing.) Let $H$ be a holomorphic square root of $h$ on $\Omega$, and let $f$ be a complex antiderivative of $H$, where we choose the constant of integration so that $f(z)$ is close to the identity in $A^{\infty}(\Omega)$. By choosing $h$ close enough to 1 in $A^{\infty}(\Omega)$, we may guarantee that $f$ is close enough to the identity that $f$ is one-to-one on $\Omega$ and $f(\Omega)$ is as $C^{\infty}$-close as we desire to $\Omega$.

Now according to Theorem 1.1, $f(\Omega)$ is a boundary arc length quadrature domain that is $C^{\infty}$ close to $\Omega$. 
We can repeat the above argument using $\mathcal{S}_{a}$ for a point $a$ in $\Omega$ instead of the complete Szegö span. We obtain a biholomorphic mapping $f$ to an arc length quadrature domain close to $\Omega$ satisfying an identity,

$$
f^{\prime}=\left(\sum_{m=0}^{N} c_{m} S^{m}(z, a)\right)^{2} .
$$

We want to see that $f^{\prime}$ is also in the Bergman span so that $f(\Omega)$ is also an area quadrature domain via Theorem 1.3 of [11].

Let $L(z, w)$ denote the Garabedian kernel associated to $\Omega$ and let $L^{m}(z, w)=$ $(\partial / \partial w)^{m} L(z, w)$. Let $\Lambda(z, w)$ denote the Schiffer kernel function, which is meromorphic in $z$ and $w$ with a double pole at $z=w$ and which is related to the Bergman kernel $K(z, w)$ of $\Omega$ via

$$
K(w, z) \overline{T(z)}=-\Lambda(w, z) T(z)
$$

when $w \in \Omega$ and $z \in b \Omega$. Here $T(z)$ denotes the complex unit tangent vector function pointing in the direction of the standard orientation. The basic properties of these kernel functions are described in [5]. We also define $\Lambda^{m}(z, w)=(\partial / \partial w)^{m} \Lambda(z, w)$. Let $b_{j}$ denote complex coefficients that will be determined later. The relationship

$$
S(w, z)=\frac{1}{i} L(z, w) T(z)
$$

for $w \in \Omega$ and $z \in b \Omega$ together with the relationship between $K(z, w)$ and $\Lambda(z, w)$ allow us to state that $H(z) T(z)=-\overline{G(z) T(z)}$ for $z$ in $b \Omega$, where

$$
H(z)=\left[\left(\sum_{m=0}^{N} c_{m} S^{m}(z, a)\right)^{2}-\left(\sum_{j=0}^{M} b_{j} K^{j}(z, a)\right)\right]
$$

and

$$
G(z)=\left[\left(\sum_{m=0}^{N} \bar{c}_{m} L^{m}(z, a)\right)^{2}-\left(\sum_{j=0}^{M} \bar{b}_{j} \Lambda^{j}(z, a)\right)\right] .
$$

It can be deduced that $\left(\sum_{j=0}^{N} \bar{c}_{j} L^{j}(z, a)\right)^{2}$ is residue free since

$$
\int_{b \Omega}\left(\sum_{m=0}^{N} \bar{c}_{m} L^{m}(z, a)\right)^{2} d z=\int_{b \Omega}\left(\sum_{m=0}^{N} c_{m} \overline{S^{m}(z, a)}\right)^{2} d \bar{z},
$$

and this last integral is zero by Cauchy's Theorem. Since $\Lambda(z, a)$ has principal part $(-1 / \pi)(z-a)^{-2}$ at $z=a$, it follows that a positive integer $M$ and constants $b_{j}$ can be chosen so that $G(z)$ has a removable singularity at $a$. Now both $H(z)$ and $G(z)$ are holomorphic on $\Omega$ and extend smoothly to the boundary in such a way that $H(z) T(z)=-\overline{G(z) T(z)}$ on the boundary. We now claim that this can only be true if both $H$ and $G$ are identically zero. Indeed, functions of the form $\overline{G(z) T(z)}$ are orthogonal to holomorphic functions in the Hardy space and functions of the form $H(z) T(z)$ are orthogonal to anti-holomorphic 
functions. Thus, $H(z) T(z)$ is orthogonal to holomorphic functions and antiholomorphic functions. But every $C^{\infty}$-smooth function on $b \Omega$ is the restriction to the boundary of a harmonic function in $C^{\infty}(\bar{\Omega})$, and every such harmonic function can be written as $h+\bar{h}$ where $h \in A^{\infty}(\Omega)$. Since smooth functions are dense in $L^{2}(b \Omega)$, it follows that $H \equiv 0$ and $G \equiv 0$. We can now state that

$$
\left(\sum_{m=0}^{N} c_{m} S^{m}(z, a)\right)^{2}=\left(\sum_{j=0}^{M} b_{j} K^{j}(z, a)\right),
$$

and this shows that $f^{\prime}$ is in the Bergman span. Hence $f$ is both a Szegö and a Bergman coordinate and $f(\Omega)$ is a double quadrature domain.

We remark here that, after finding the proof above, we discovered that Avci [2] showed in his unpublished Stanford $\mathrm{PhD}$ thesis using other methods that an arc length quadrature domain that satisfies a one point quadrature identity would also have to be an area quadrature domain. We include our proof here because it shows the close and explicit connection between Szegö and Bergman coordinates, and because we will improve upon it in $\$ 5$ when we generalize our results to smooth multiply connected domains.

We now turn to the simple proof of density of double quadrature domains in the simply connected setting promised at the beginning of this section. The proof is based on a theorem of Aharonov and Shapiro for area quadrature domains and a theorem of Shapiro and Ullemar for arc length quadrature domains. Let $\Omega$ be a bounded simply connected domain bounded by a $C^{\infty}$-smooth Jordan curve and let $f$ denote a Riemann mapping of $\Omega$ to the unit disc. Let $F=f^{-1}$. Note that a branch of $\sqrt{F^{\prime}}$ can be chosen which is analytic on the unit disc and which extends to be in $A^{\infty}\left(D_{1}(0)\right)$. Aharonov and Shapiro [1] proved that $\Omega$ is an area quadrature domain if and only if $F$ is rational. Shapiro and Ullemar [21] proved that $\Omega$ is an arc length quadrature domain if and only if $F^{\prime}$ is the square of a rational function. To construct a double quadrature domain that is $C^{\infty}$ close to $\Omega$, we may approximate $\sqrt{F^{\prime}}$ in $A^{\infty}\left(D_{1}(0)\right)$ by a polynomial $P(z)$. Let $p(z)$ be (a polynomial) antiderivative of $P(z)^{2}$ where the constant of integration is chosen so that $p(z)$ is close to $F^{\prime}$ in $A^{\infty}\left(D_{1}(0)\right)$. If the first polynomial approximation $P(z)$ is sufficiently close to $\sqrt{F^{\prime}}$, then the image of the unit disc under $p(z)$ will be a double quadrature domain that is $C^{\infty}$ close to $\Omega$.

\section{Density of Boundary ARC Length QuAdrature Domains in The MULTIPLY CONNECTED CASE}

In this section, we show how the argument in [15, p. 77-78] using half-order differentials and a special Runge Theorem on Riemann surfaces can be modified along the lines of the previous section to prove $C^{\infty}$ density of boundary arc length quadrature domains in the category of smooth finitely connected domains.

Let $\Omega$ be a bounded domain bounded by $n$ non-intersecting $C^{\infty}$-smooth Jordan curves. There is a function $h$ in the Szegö span that is as close to one in $C^{\infty}(\bar{\Omega})$ as we desire. We will need the rational functions $R_{j}(z), j=1, \ldots, n-1$ constructed 
in [15, p. 78] that satisfy the following special orthogonality conditions. Let $\gamma_{j}$, $j=0, \ldots, n-1$ denote the boundary curves of $\Omega$ where $\gamma_{0}$ denotes the outer boundary. Choose a point $z_{j}$ inside $\gamma_{j}, j=1, \ldots, n-1$ (i.e., choose one point from each of the holes in $\Omega$ ). The rational functions are given by

$$
R_{j}(z)=\frac{1}{2 \pi i\left(z-z_{j}\right)}+\left(z-z_{j}\right) Q_{j}(z)
$$

where the $Q_{j}$ are any polynomials satisfying

$$
Q_{j}\left(z_{k}\right)=-\frac{1}{2 \pi i\left(z_{k}-z_{j}\right)^{2}}
$$

for $k \neq j$ (making $R_{j}\left(z_{k}\right)=0$ for $k \neq j$ ). The functions $R_{j}$ have the property that

$$
\int_{\gamma_{k}} R_{j} d z=\delta_{k j} \quad \text { and } \int_{\gamma_{k}} R_{j} R_{m} d z=0
$$

for $j, k, m=1, \ldots n-1$. Since each rational function $R_{j}$ is in $A^{\infty}(\Omega)$, we may approximate it in $A^{\infty}(\Omega)$ by a function $r_{j}$ in the Szegö span. Notice that it easy to find coefficients $A_{j}$ such that the periods of $\left(h-\sum_{j=1}^{n-1} A_{j} R_{j}\right)^{2}$ vanish because the system is

$$
\int_{\gamma_{k}} h^{2} d z+2 \sum_{j=1}^{n-1} A_{j} \int_{\gamma_{k}} h R_{j} d z=0
$$

for $k=1, \ldots, n-1$. Since $h$ is close to one, the coefficient matrix

$$
\int_{\gamma_{k}} h R_{j} d z
$$

is close to the identity and $\int_{\gamma_{k}} h^{2} d z$ is close to zero. Thus, the $A_{j}$ are uniquely determined and are close to zero. If we modify the problem of making the periods vanish by replacing the $R_{j}$ by our functions $r_{j}$ in the Szegö span, then the system becomes

$$
\int_{\gamma_{k}} h^{2} d z+2 \sum_{j=1}^{n-1} A_{j} \int_{\gamma_{k}} h r_{j} d z+\sum_{j, m=1}^{n-1} A_{j} A_{m} \int_{\gamma_{k}} r_{j} r_{m} d z=0,
$$

and since the coefficients in the linear part are close to the identity matrix and the coefficients of the quadratic terms are small, the implicit function theorem yields that the $A_{j}$ are uniquely determined and are close to the small solution to the system with $R_{j}$ in place of $r_{j}$. Notice that by taking $h$ to be sufficiently close to one, the coefficients $A_{j}$ can be made small enough that $h-\sum_{j-1}^{n-1} A_{j} r_{j}$ is as close to one as desired. Thus, this shows that there is a function $F=h-\sum_{j-1}^{n-1} A_{j} r_{j}$ in the Szegő span which is close to one whose square $F^{2}$ has vanishing periods. Now let $f$ be an antiderivative of $F^{2}$. Since $F^{2}$ is close to one, we may choose the constant of integration so that $f$ is close to the identity. If $h$ is close enough to one, then $f$ will be close enough to the identity to make $f$ biholomorphic on $\Omega$ and Theorem 1.1 yields that $f$ maps $\Omega$ to a boundary arc length quadrature domain that is $C^{\infty}$ close to $\Omega$. 


\section{Density of Double Quadrature domains in the General CASE}

We continue with the same domain and setup as in the previous section. In particular, $\gamma_{j}, j=0, \ldots, n-1$ denote the boundary curves of $\Omega$ where $\gamma_{0}$ denotes the outer boundary. Let $p=n-1$, and let $\widehat{\Omega}$ denote the double of $\Omega$. We are now thinking of the curves $\gamma_{j}$ as being curves on $\widehat{\Omega}$. Define $p$ more curves $\gamma_{j}$, $j=p+1, \ldots, 2 p$, on $\widehat{\Omega}$ as follows. Let $\Gamma_{j}$ be a simple smooth curve that starts at a point on $\gamma_{j}$, enters $\Omega$, and terminates at a point on the outer boundary $\gamma_{0}$. We may choose the $\Gamma_{j}$ so that no two of them intersect. In this case, the domain $U$ given by $\Omega-\cup_{j=1}^{p} \Gamma_{j}$ is a simply connected domain. Let $\widetilde{\Gamma}_{j}$ denote the curve which is the image of the reverse of $\Gamma_{j}$ in the reflected copy of $\Omega$ in $\widehat{\Omega}$, and let $\gamma_{p+j}$ be the closed curve in $\widehat{\Omega}$ obtained by following $\Gamma_{j}$ in $\Omega$ and then $\widetilde{\Gamma}_{j}$ in the reflected copy of $\Omega$ back to the starting point. In this way, we obtain a standard homology basis, $\gamma_{j}, j=1, \ldots, 2 p$, for $\widehat{\Omega}$ where the first $p$ curves are the standard homology basis for $\Omega$ and the last $p$ curves wrap around the "handles" of $\widehat{\Omega}$ and complete the homology basis for $\Omega$ to a homology basis for $\widehat{\Omega}$.

We will need the concept of a half-order differential on the double as used in [15]. In the present context, we will describe a meromorphic half-order differential $h$ on $\widehat{\Omega}$ as a pair of meromorphic functions $\left(h_{1}, h_{2}\right)$ where the two functions are meromorphic on $\Omega$, extend continuously to the boundary (and are allowed to take the value $\infty$ at a boundary point), and satisfy

$$
h_{1}(z) T(z)=\overline{h_{2}(z)}
$$

on the boundary of $\Omega$. The symbol $h$ stands for the pair $\left(h_{1}, h_{2}\right)$. These objects have the virtue that identity (5.1) allows the meromorphic differential $h_{1}{ }^{2} d z$ on $\Omega$ to be extended to a meromorphic differential on $\widehat{\Omega}$ (via extension by $\overline{h_{2}{ }^{2}} d \bar{z}$ on the anti-holomorphic reflected side). We let $h^{2} d z$ denote the extension of $h_{1}^{2} d z$ to the double. Another important feature is that if $h=\left(h_{1}, h_{2}\right)$ and $g=\left(g_{1}, g_{2}\right)$ represent two half-order differentials, then $h_{1} g_{1} d z$ extends meromorphically to $\widehat{\Omega}$ as a meromorphic differential (via extension by $\overline{h_{2} g_{2}} d \bar{z}$ on the reflected side). We let $h g d z$ denote the extension of this form to the double. Hence, if $\gamma$ is a curve on $\widehat{\Omega}$ and $h=\left(h_{1}, h_{2}\right)$ and $g=\left(g_{1}, g_{2}\right)$ are half-order differentials, we may define the pairing

$$
B(h, g)=\int_{\gamma} h g d z
$$

as long as the extension $h g d z$ to $\widehat{\Omega}$ does not have poles along the curve.

Notice that identity (5.1) is the same as the critical identity (3.1) for the Szegö and Garabedian kernels. Results in [7] (Lemma 6.1) show that all meromorphic half-order differentials without poles on the boundary arise as linear combinations of the Szegö and Garabedian kernels and their derivatives in the second variable. When there are no poles inside the domain, a meromorphic half-order differential must be a linear combination of just the Szegö kernel and its derivatives in the second variable on the domain side. This is the key connection between the Szego" 
kernel and the results from [15] that allow us to use Szegö coordinates to prove the density of double quadrature domains here.

The idea of the proof is to construct a half-order differential $h=\left(h_{1}, h_{2}\right)$ with the property that $h_{1}$ is in the Szegó span and is close to one in $A^{\infty}(\Omega)$, and $h^{2} d z$ is a meromorphic one-form on $\widehat{\Omega}$ with a single pole at a point in the reflected side of $\Omega$ in $\widehat{\Omega}$ with the property that $\int_{\gamma_{j}} h^{2} d z=0$ for $j=1, \ldots, 2 p$. Then $h^{2} d z=d f$ where $f$ is a meromorphic function on $\widehat{\Omega}$. If $h_{1}$ is sufficiently close to one, then the condition $f^{\prime}=h_{1}^{2}$ implies that $f$ is a biholomorphic mapping of $\Omega$ onto a domain which is $C^{\infty}$ close to $\Omega$. Now Theorem 1.1 yields that $f(\Omega)$ is a boundary arc length quadrature domain. Finally, by [14], the fact that $f$ extends meromorphically to the double yields that $f(\Omega)$ is an area quadrature domain. In this way, we will obtain a double quadrature domain that is $C^{\infty}$ close to $\Omega$.

We shall need the following special Mergelyan approximation lemma. Suppose $a_{0} \in U$. Recall that $\mathcal{S}_{a_{0}}$ denotes the complex linear span of functions of $z$ from $\left\{S^{m}\left(z, a_{0}\right): m=0,1,2, \ldots\right\}$.

Lemma 5.1. Given a holomorphic function $G$ in $A^{\infty}(\Omega)$ and continuous functions $\varphi_{j}$ on $\Gamma_{j}$ that satisfy the compatibility condition

$$
G(w) T(w)=\overline{\varphi_{j}(w)}
$$

at the endpoints $w$ of each curve $\Gamma_{j}$ (which fall on $b \Omega$ ), it is possible to find a function

$$
\sigma(z)=\sum_{m=0}^{N} c_{m} S^{m}\left(z, a_{0}\right)
$$

in $\mathcal{S}_{a_{0}}$ such that $\sigma$ is as close as desired to $G$ in $A^{\infty}(\Omega)$ and so that

$$
\lambda(z)=-i \sum_{m=0}^{N} \overline{c_{m}} L^{m}\left(z, a_{0}\right)
$$

is as close to $\varphi_{j}$ on each $\Gamma_{j}$ in the uniform topology as desired. (Note that identity (3.1) shows that $(\sigma, \lambda)$ is a half-order differential.)

We will now prove the density of double quadrature domains, assuming the truth of the approximation lemma. Afterwards, we will prove Lemma 5.1.

Let $K$ denote the compact set $\bar{\Omega} \cup\left(\cup_{j=1}^{p} \gamma_{j+p}\right)$ in $\widehat{\Omega}$. We may consider nonmeromorphic half-order differentials defined on $K$ where $g=\left(g_{1}, g_{2}\right)$ is such that $g_{1}$ is in $C^{\infty}(\bar{\Omega})$ and the $g_{2}$ is a continuous function on the union of the curves $\Gamma_{j}$ and satisfies the compatibility condition

$$
g_{1}(w) T(w)=\overline{g_{2}(w)}
$$

at the endpoints $w$ of each curve $\Gamma_{j}$. For two such half-order differentials $g$ and $h$, we may define the pairing

$$
B_{k}(g, h)=\int_{\gamma_{k}} g h d z
$$


for $k=1, \ldots, 2 p$ as described above in the meromorphic case.

We now define $2 p+1$ such half-order differentials $g^{m}=\left(g_{1}^{m}, g_{2}^{m}\right), m=0, \ldots, 2 p$, on $K$ satisfying

(i) $g_{1}^{0}=1$ on $\bar{\Omega}$,

(ii) $B_{k}\left(g^{0}, g^{0}\right)=0$ for all $k=1, \ldots, 2 p$, and

(iii) $B_{k}\left(g^{0}, g^{m}\right)=\delta_{k m}$ for $k, m=1, \ldots, 2 p$,

We start by setting $g_{1}^{0} \equiv 1$ on $\bar{\Omega}$ (where $g_{1}^{0}$ is the first function associated to the half-order differential $g^{0}$ ) as condition (ii) dictates. Notice that (iii) is therefore automatically satisfied for $k=1, \ldots, p$. It is now an easy matter to define $g_{2}^{0}$ on the curves $\Gamma_{j}$ so that the compatibility conditions hold and condition (iii) holds for $k=p+1, \ldots, 2 p$ as well. Next, set $g_{1}^{m}=R_{m}$ on $\bar{\Omega}$, where $R_{m}$ are the rational functions of the previous section for $m=1, \ldots, p$. Note that condition (iii) now holds for $k, m=1, \ldots, p$. It is another simple matter to define $g_{2}^{m}$ on the curves $\Gamma_{j}$ so that (iii) also holds for $k=p+1, \ldots, 2 p$ and $m=1, \ldots, p$. Finally, $g^{m}$ are chosen for $m=p+1, \ldots, 2 p$ so that $g_{1}^{m} \equiv 0$ on $\bar{\Omega}$ and $g_{2}^{m}$ are defined on the curves $\Gamma_{j}$ so that (iii) holds for $k=p+1, \ldots, 2 p$. Note that (iii) holds automatically for $k=1, \ldots, p$.

Lemma 5.1 implies that there are half-order differentials $h^{m}=\left(h_{1}^{m}, h_{2}^{m}\right)$ which are close to the $g^{m}$ in the sense that $h_{1}^{m}$ is close to $g_{1}^{m}$ in $A^{\infty}(\Omega)$ for each $m$ and $h_{2}^{m}$ is uniformly close to $g_{2}^{m}$ on the curves $\Gamma_{j}$. The half-order differential that we seek will be given by

$$
h=h^{0}+\sum_{m=1}^{2 p} t_{m} h^{m},
$$

where the $t_{m}$ are complex numbers close to zero. (Note that we may take linear combinations of half-order differentials in the obvious way by summing the corresponding functions in the pairs.) We will choose the $t_{m}$ to make $B_{k}(h, h)=0$ for $k=1, \ldots, 2 p$ so that $h^{2} d z$ is period free, and is therefore an exact meromorphic differential $d f$. (Note that the lone pole of $h^{2} d z$ would necessarily have residue equal to zero.) Let $g=g^{0}+\sum_{m=1}^{2 p} t_{m} g^{m}$. Define complex coefficients via

$$
\begin{aligned}
a_{k i j} & =B_{k}\left(h^{i}+g^{i}, h^{j}-g^{j}\right) \\
b_{k j} & =2\left(B_{k}\left(g^{j}, h^{0}-g^{0}\right)+B_{k}\left(h^{0}, h^{j}-g^{j}\right)\right) \\
c_{k} & =B_{k}\left(h^{0}+g^{0}, h^{0}-g^{0}\right) \\
A_{k i j} & =B_{k}\left(g^{i}, g^{j}\right)
\end{aligned}
$$

Note that $A_{k i j}$ is fixed, while the others depend on the $h^{j}$ and can be made arbitrarily small by improving the approximation sufficiently. Expand $B_{k}(h, h)=$ $B_{k}(h-g+g, h-g+g)$ to obtain

$$
B_{k}(h, h)=2 t_{k}+c_{k}+\sum_{i, j} A_{k i j} t_{i} t_{j}+\sum_{i, j} a_{k i j} t_{i} t_{j}+\sum_{j} b_{k j} t_{j} .
$$

We obtain a system by setting these $2 p$ periods equal to zero. Note that this system has the unique zero solution if the coefficients that depend on the $h^{j}$ 
are taken to be zero. It is now a standard application of the implicit function theorem to deduce that it is possible to choose $t_{j}$ close to zero to make all these periods vanish. This completes the proof, assuming Lemma 5.1. We now turn to proving the lemma.

Note that Theorem 1.2 allows us to simplify the assumptions in Lemma 5.1 by subtracting off an element of the Szegö span to be able to assume that $G$ is zero on $\bar{\Omega}$. Given the point $a_{0}$ in the simply connected domain $U=\Omega-\cup_{j=1}^{p} \Gamma_{j}$, our task then is to find a function $\lambda(z)$ which is a linear combination of functions of the form $L^{m}\left(z, a_{0}\right)$ which is uniformly close to the functions $\varphi_{j}$ on each $\Gamma_{j}$ and which is close in $C^{\infty}(b \Omega)$ to zero. This turns out to be remarkably easy. The first step in the argument is to use the classical Mergelyan Theorem (see Rudin [18], Chapter 20, Exercise 1) and Runge's Theorem to approximate our functions as follows. For $\epsilon>0$, let $V_{\epsilon}$ denote the points in $\mathbb{C}$ that are less than or equal to a distance $\epsilon$ from $b \Omega$, i.e., the closure of a small collared neighborhood of the boundary in $\mathbb{C}$. Let $K_{\epsilon}=V_{\epsilon} \cup\left(\cup_{j=1}^{p} \Gamma_{j}\right)$. We first use Mergelyan's Theorem to obtain a rational function $R(z)$ with poles in $\mathbb{C}-K_{\epsilon}$ that is unformly close to zero in $V_{\epsilon}$, and because the functions $\varphi_{j}$ vanish on $b \Omega$, and because we may shrink $\epsilon$, we may also arrange for $R(z)$ to be uniformly close to the $\varphi_{j}$ on each $\Gamma_{j}$. Cauchy's Estimates reveal that uniform convergence on the interior of $V_{\epsilon}$ implies $C^{\infty}$ convergence on $b \Omega$. Hence, we may obtain a rational function that is close to zero in $C^{\infty}(b \Omega)$ and uniformly close to $\varphi_{j}$ on each $\Gamma_{j}$. Next, we may slide the poles of $R(z)$ that fall in $U$ to the point $a_{0}$ as is done in many standard proofs of Runge's Theorem (see Stein [22, p. 63]) to obtain a rational function $R(z)$ with poles at $a_{0}$ and finitely many other points outside of $\bar{\Omega}$ which is as close in $C^{\infty}(b \Omega)$ to zero as desired and as close in the uniform topology to $\varphi_{j}$ on each $\Gamma_{j}$ as desired.

The Garabedian kernel $L(z, w)$ is such that

$$
L(z, w)=\frac{1}{2 \pi(z-w)}+\ell(z, w),
$$

where $\ell(z, w)$ is holomorphic in $z$ and $w$ and is in $C^{\infty}(\bar{\Omega} \times \bar{\Omega})$ (see [5, p. 102]). We will therefore be able to use $L(z, w)$ much like a Cauchy kernel in what follows. The Residue Theorem yields that, for $z \in \Omega-\left\{a_{0}\right\}$,

$$
\frac{1}{i} \int_{w \in b \Omega} L(z, w) R(w) d w
$$

is equal to $R(z)$ plus $2 \pi$ times the residue of $L(z, w) R(w)$ at $a_{0}$, which is a fixed finite linear combination of functions of the form $L^{m}\left(z, a_{0}\right)$. If we show that the integral tends to zero in $z$ in $A^{\infty}(\Omega)$, then we will have completed the proof. This turns out to be an easy consequence of the fact that the Szegö projection is a continuous linear operator in $C^{\infty}(\bar{\Omega})$ (see [5, p. 13]). Indeed, identity (3.1) and the fact that $L(z, w)=-L(w, z)$ yields that the integral is equal to

$$
-\int_{w \in b \Omega} S(z, w) R(w) d s
$$


which is the Szegö projection evaluated at $z$ of the function which is the restriction of $R(w)$ to the boundary. Since $R$ is close to zero in $C^{\infty}(b \Omega)$, it follows that the function given by the integral is close to zero in $A^{\infty}(b \Omega)$. The proof of the lemma is finished.

\section{Properties of Double quadrature domains}

Suppose $\Omega$ is a double quadrature domain in the plane. Because $\Omega$ is an area quadrature domain, it has a boundary given by an algebraic curve, and because it is a boundary arc length quadrature domain, that curve consists of $C^{\infty}$ smooth real analytic curves (see [1, 14, 15]). Because $\Omega$ is an area quadrature domain, the complex polynomials belong to the Bergman span (see [11]). Also, the Schwarz function $S(z)$ exists and is meromorphic on $\Omega$, extends analytically past the boundary, and satisfies $S(z)=\bar{z}$ on the boundary (see [1, 14]). This identity shows that the two functions $z$ and $S(z)$ extend meromorphically to the double of $\Omega$. It was proved in [14] that the extensions form a primitive pair for the field of meromorphic functions on the double, i.e., all the meromorphic functions on the double are given as rational combinations of the two. It is shown in [8] (see also [9]) that the Bergman kernel $K(z, w)$ associated to an area quadrature domain

is a rational function of $z, S(z)$, and $\bar{w}$, and $\overline{S(w)}$. Consequently, $K(z, w)$ is a rational function of $z, \bar{z}, w$, and $\bar{w}$ when $z$ and $w$ are restricted to the boundary. It was also shown in [8, 9] that the Bergman kernel is a rational combination of two Ahlfors maps associated to two generic points in the domain. Aharonov and Shapiro [1] showed that the Ahlfors maps are algebraic.

Because $\Omega$ is an arc length quadrature domain, there is a meromorphic function $H(z)$ on $\Omega$ which extends analytically past the boundary and such that $\overline{H(z)}$ is equal to the complex unit tangent vector function $T(z)$ on the boundary (see [15, 2]). The identity relating the Szegő and Garabedian kernels can be used with this fact to see that both the Szegö and the Garabedian kernels extend to the double in the first variable when the second variable is held fixed. Indeed, the identity

$$
\overline{S(z, a)}=\frac{1}{i} L(z, a) T(z) \quad \text { for } z \in b \Omega
$$

shows that $L(z, a)=i \overline{S(z, a)} / \overline{H(z)}$ on the boundary, and this shows that $L(z, a)$ extends meromorphically to the double in $z$. Similarly, $S(z, a)$ extends. These identities also reveal that $T(z)$ is equal to a function $G(z)$ on the boundary where $G(z)$ is meromorphic on $\Omega$ and extends analytically past the boundary. Hence, $T(z)$ is the restriction to the boundary of a meromorphic function on the double of $\Omega$ that has no singularities on the boundary of $\Omega$. These arguments are reversible and we may state the following theorem.

Theorem 6.1. A bounded domain with a piecewise $C^{1}$ smooth boundary is a boundary arc length quadrature domain if and only if the Szegö kernel $S(z, w)$ associated to the domain extends meromorphically to the double as a function of $z$ for each fixed $w$ in the domain. This is the case if and only if the complex 
unit tangent vector function is the restriction to the boundary of a meromorphic function that has no singularities on the boundary of $\Omega$.

The same theorem holds with the Garabedian kernel in place of the Szegö kernel. (The part of the theorem about $T$ was proved in [15]. The equivalence of the extendibility of the Szegö kernel is a rather direct consequence of the extendibility of $T$ and might very well have been noted earlier.)

If $\Omega$ is a double quadrature domain, then we have shown that $T(z)$ is the restriction to the boundary of a meromorphic function on the double, and we also know that such functions are generated by $z$ and $S(z)$. Hence, it follows that $T(z)$ is the restriction of a rational combination of $z$ and $S(z)$ to the boundary, i.e., $T(z)$ is a rational function of $z$ and $\bar{z}$.

When Theorem 6.1 is combined with results from [6], it follows that the Szegő kernel $S(z, w)$ associated to a double quadrature domain is a rational combination of $z, S(z)$, and $\bar{w}$, and $\overline{S(w)}$. Consequently, $S(z, w)$ is a rational function of $z$, $\bar{z}, w$, and $\bar{w}$ when $z$ and $w$ are restricted to the boundary.

The Kerzman-Stein kernel is given by

$$
A(z, w)=\frac{1}{2 \pi i}\left(\frac{T(w)}{w-z}-\frac{\overline{T(z)}}{\bar{w}-\bar{z}}\right)
$$

for $z$ and $w$ in the boundary of $\Omega$, and it follows that the Kerzman-Stein kernel associated to a double quadrature domain is a rational function of $z, \bar{z}, w$, and $\bar{w}$.

We shall now show that the complex polynomials are in the Szegö span associated to a double quadrature domain. Let $H(z)$ denote the meromorphic function that is equal to $\overline{T(z)}$ on the boundary. Suppose $h \in A^{\infty}(\Omega)$. Since $\bar{z}=S(z)$ on the boundary, we may write

$$
\int_{b \Omega} h \bar{z}^{n} d s=\int_{b \Omega} h(z) S(z)^{n} \overline{T(z)} d z=\int_{b \Omega} h(z) S(z)^{n} H(z) d z,
$$

and this last integral is equal to a finite linear combination of values of $h$ and its derivatives at finitely many points in $\Omega$ by the Residue Theorem. Hence, $z^{n}$ has the same effect when paired with $h$ that a certain element in the Szegö span would have. Since $A^{\infty}(\Omega)$ is dense in the Hardy space, it follows that $z^{n}$ is equal to the element in the Szegö span.

The converse of this last result is true, namely, that if the complex polynomials are in the Szegő span, then the domain is a double quadrature domain. To see this, note that $z=z / 1$. If both $z$ and 1 are in the Szegö span, then $z$ is a quotient of elements in the Szegö span, and as such, it extends to the double as a meromorphic function (since the relationship between the Szegö and the Garabedian kernels reveals that quotients of functions in the Szegö span would be equal to quotients of conjugates of functions in the "Garabedian span" on the boundary). The condition that $z$ extends to the double is the classic condition that is equivalent to the domain being an area quadrature domain. It is easy to 
see that a smooth domain is a boundary arc length quadrature domain if and only if the constant function 1 is in the Szegö span. Hence, we have proved the following theorem.

Theorem 6.2. A bounded domain with a piecewise $C^{1}$ smooth boundary is a double quadrature domain if and only if the complex polynomials belong to the Szegö span associated to the domain.

Theorem 6.2 is an appealing analogue of Theorem 1.2 from [1] which states that a bounded domain is an area quadrature domain if and only if the complex polynomials belong to the Bergman span.

The Poisson kernel of a smooth simply connected domain is given by

$$
p(z, w)=2 \operatorname{Re}\left(\frac{S(z, w) S(w, a)}{S(a, a)}\right)-\frac{|S(w, a)|^{2}}{S(a, a)},
$$

where $z \in \Omega, w \in b \Omega$, and $a$ is a fixed point in $\Omega$ (see [3, p. 1367]). Hence, when $\Omega$ is a simply connected double quadrature domain, $p(z, w)$ is the real part of a function that is rational in $z$ and $S(z)$, and $w$ and $\bar{w}$ (just like in the unit disc, where $S(z)=1 / z)$.

The rest of the properties mentioned in $\S 1$ involving the Riemann map $f$ of a simply connected domain follow from the remarks above and the fact that $f$ extends meromorphically to the double and the extension generates the field of meromorphic functions on the double, i.e., every meromorphic function on the double is a rational function of the extension of the Riemann map. Hence, for example, on a quadrature domain with respect to area, the functions $z$ and $S(z)$ extend to the double, and so they are rational functions of $f(z)$. Since, $f(w)=1 / \overline{f(w)}$ on the boundary, the restriction to the boundary of a rational function of $f(w)$ and $\overline{f(w)}$ is equal to a rational function of $f(w)$ alone. Hence, it follows from the remarks above about the Poisson kernel is the real part of a rational function of $f(z)$ and $f(w)$.

\section{The SchWARZ Function AND PROPER Holomorphic MAPpings to the DISC}

We conclude this paper by noting a relationship between proper holomorphic mappings of a domain to the unit disc and the existence of the Schwarz function, which is the hallmark of a bounded quadrature domain with respect to area. We go on to reveal a further relationship between proper holomorphic mappings and the extension of the unit tangent function to the double, which characterizes a bounded quadrature domain with respect to area.

Assume that $\Omega$ is a (bounded) area quadrature domain. For the moment, assume that zero does not belong to $\Omega$. As remarked earlier, the Schwarz function $S(z)$ exists and is meromorphic in $\Omega$. Let $\left\{a_{j}\right\}_{j=1}^{N}$ denote the poles of $S(z)$ in $\Omega$, and suppose $a_{j}$ is a pole of $S(z)$ of order $n_{j}$. Let $f_{j}$ denote the Ahlfors map associated to $a_{j}$, which is a proper holomorphic mapping of $\Omega$ onto the 
unit disc such that $a_{j}$ is a simple zero of $f_{j}$. (The Ahlfors map associated to a point $a$ in $\Omega$ is the unique solution to the extremal problem to maximize $h^{\prime}(a)$ under the conditions that $h$ maps $\Omega$ into the unit disc and $h^{\prime}(a)$ is real. It maps a bounded $n$-connected domain onto the unit disc as an $n$-to-one branched covering map.) We note here that, since the boundary of $\Omega$ is piecewise smooth, proper holomorphic mappings to the unit disc extend continuously to the boundary. Conversely, a holomorphic mapping to the unit disc that is continuous up to the boundary is proper if and only if it is non-constant and it maps the boundary to the unit circle. Notice that, since $\left|f_{j}(z)\right|=1$ and $\bar{z}=S(z)$ on the boundary, the function

$$
\Phi(z)=\left(\prod_{j=1}^{N} f_{j}(z)^{n_{j}}\right) \frac{S(z)}{z}
$$

has removable singularities at each $a_{j}$, is continuous up to the boundary, and has unit modulus on the boundary. If $\Omega$ is multiply connected, then $\Phi$ must have a zero in $\Omega$ since the Schwarz function must have a zero in the domain (see [2]). Hence $\Phi$ is a proper holomorphic mapping of $\Omega$ to the unit disc. It follows that $S(z)$ is $z$ times the quotient of two proper holomorphic mappings of $\Omega$ to the unit disc. If the point zero were in the domain, we could add the Ahlfors map $f_{0}$ to the product and obtain the same result. If $\Omega$ is simply connected, then we can define

$$
\Psi(z)=\left(\prod_{j=1}^{N} f_{j}(z)^{n_{j}}\right)\left(\prod_{k=1}^{M} F_{k}(z)^{-m_{k}}\right) \frac{S(z)}{z},
$$

where $f_{j}$ is the Riemann map associated to a point $a_{j}$ where $S(z)$ has a pole of order $n_{j}$, and $F_{k}$ is the Riemann map associated to a point $b_{k}$ where $S(z)$ has a zero of order $m_{k}$. In this case, $\Psi$ has constant modulus one on the boundary and no zeros in $\Omega$. Hence it is a unimodular constant and we deduce that $S(z)$ is equal to a unimodular constant times $z$ times a quotient of products of Riemann maps. Since proper maps are all given by unimodular constants times products of Riemann maps in this setting, the same result follows, i.e., that $S(z)$ is $z$ times the quotient of two proper holomorphic mappings of $\Omega$ to the unit disc.

If the domain $\Omega$ is bounded by finitely many non-intersecting Jordan curves, a simple converse follows. Indeed, since a proper holomorphic mapping $f$ to the unit disc would be continuous up to the boundary and have unit modulus on the boundary in this case, the identity $f(z)=1 / \overline{f(z)}$, which holds on the boundary, reveals that $f$ extends to the double. If $\bar{z}$ is equal to $z$ times the quotient of two proper holomorphic mappings to the unit disc on the boundary, then $z$ is seen to extend to the double, and it follows that the domain is an area quadrature domain. The theorem with its converse reads as follows.

Theorem 7.1. A bounded domain in the plane bounded by finitely many nonintersecting Jordan curves is an area quadrature domain if and only if, on the boundary, the function $\bar{z} / z$ is equal to the boundary values of a quotient of proper holomorphic mappings of the domain onto the unit disc. 
Note that if $\bar{z} / z=f_{1} / f_{2}$ on the boundary, then the Schwarz function is given by $S(z)=z f_{1}(z) / f_{2}(z)$.

If a domain has piecewise $C^{1}$ smooth boundary, it is a boundary arc length quadrature domain if and only if the unimodular function $T(z)$ extends to the double as a meromorphic function. Similar reasoning to the argument above with $T(z)$ in place of $S(z) / z$ yields the following result.

Theorem 7.2. A finitely connected bounded domain with piecewise $C^{1}$ smooth boundary is a boundary arc length quadrature domain if and only if the complex unit tangent vector function $T(z)$ is equal to a quotient of proper holomorphic mappings of the domain onto the unit disc.

Since an area quadrature domain has piecewise $C^{1}$ smooth boundary, we may also state this last theorem.

Theorem 7.3. A bounded domain in the plane bounded by finitely many nonintersecting Jordan curves is a double quadrature domain if and only if, on the boundary, the function $\bar{z} / z$ is equal to the boundary values of a quotient of proper holomorphic mappings of the domain onto the unit disc, and the complex unit tangent vector function $T(z)$ is also equal to the boundary values of a quotient of proper holomorphic mappings of the domain onto the unit disc.

Finally, we remark that the semi-group of all proper holomorphic mappings of a finitely connected domain to the unit disc has been described completely in [12].

\section{REFERENCES}

[1] Aharonov, D. and H. S. Shapiro, Domains on which analytic functions satisfy quadrature identities, Journal D'Analyse Mathématique 30 (1976), 39-73.

[2] Avci, Y., Quadrature identities and the Schwarz function, Stanford University PhD thesis, 1977.

[3] Bell, S., Solving the Dirichlet problem in the plane by means of the Cauchy integral, Indiana Math. J. 39 (1990), 1355-1371.

[4] Bell, S., Unique continuation theorems for the $\bar{\partial}$-operator and applications, J. of Geometric Analysis 3 (1993), 195-224.

[5] Bell, S., The Cauchy transform, potential theory, and conformal mapping, CRC Press, Boca Raton, 1992.

[6] Bell, S., The fundamental role of the Szegö kernel in potential theory and complex analysis, J. für die reine und angewandte Mathematik 525 (2000), 1-16.

[7] Bell, S., Complexity in complex analysis, Advances in Math. 172 (2002), 15-52.

[8] Bell, S., Quadrature domains and kernel function zipping, Arkiv för Matematik 43 (2005), 271-287.

[9] Bell, S., The Bergman kernel and quadrature domains in the plane, Operator Theory: Advances and Applications 156 (2005), 35-52.

[10] Bell, S., Bergman coordinates, Studia Math. 176 (2006), 69-83.

[11] Bell, S., Density of quadrature domains in one and several complex variables, Complex Variables and Elliptic Equations 54 (2009), 165-171.

[12] Bell, S. and Kaleem F., The structure of the semigroup of proper holomorphic mappings of a planar domain to the unit disc, Computational Methods and Function Theory 8 (2008), 225-242. 
[13] Ebenfelt, P., B. Gustafsson, D. Khavinson, and M. Putinar, Quadrature domains and their applications, Operator Theory: Advances and Applications 156, Birkhäuser, Basel, 2005.

[14] Gustafsson, B., Quadrature domains and the Schottky double, Acta Applicandae Math. 1 (1983), 209-240.

[15] Gustafsson, B., Applications of half-order differentials on Riemann surfaces to quadrature identities for arc-length, Journal D'Analyse Math. 49 (1987), 54-89.

[16] Gustafsson, B., A distortion theorem for quadrature domains for harmonic functions, J. Math. Anal. Appl. 202 (1996), 169-182.

[17] Gustafsson, B. and H. Shapiro, What is a quadrature domain? Operator Theory: Advances and Applications 156 (2005), 1-25.

[18] Rudin, W., Real and Complex Analysis, McGraw Hill, New York, 1987.

[19] Sakai, M., Linear combinations of harmonic measures and quadrature domains of signed measures with small supports, Proc. Edinburgh Math. Soc. 42 (1999), 433444.

[20] Shapiro, H. S., The Schwarz function and its generalization to higher dimensions, Univ. of Arkansas Lecture Notes in the Mathematical Sciences, Wiley, New York, 1992.

[21] Shapiro, H. and C. Ullemar, Conformal mappings satisfying certain extremal properties and associated quadrature identities, Research Report TRITA-MAT-1986-6, Royal Inst. of Technology, 40 pp., 1981.

[22] Stein, E. M. and R. Shakarchi, Complex analysis, Princeton Lectures in Analysis II, Princeton Univ. Press, Princeton, 2003.

Mathematics Department, Purdue University, West Lafayette, IN 47907

E-mail address: bell@math.purdue.edu

Department of Mathematics, KTH, 10044 Stockholm, Sweden

E-mail address: gbjorn@kth.se

Mathematics Department, University of California Berkeley, CA 94720

E-mail address: zack@math.berkeley.edu 